\title{
HEARING DISORDERS - PART OF FETAL ALCOHOL SYNDROME
}

\author{
Georgiana Russu', Cristina Rusu², Alexandra Grosu ${ }^{3}$ \\ ${ }^{1}$ Pediatric Cardiology Clinic, "Sf. Maria" Children's Emergency Hospital, Iasi \\ ${ }^{2}$ Genetics Department, "Gr. T. Popa” University of Medicine and Pharmacy, Iasi \\ ${ }^{3}$ Pediatric resident, "Sf. Maria" Children's Emergency Hospital, Iasi
}

\begin{abstract}
Fetal alcohol syndrome (FAS) (1-2/1000 newborns), resulting from maternal consumption of alcohol early in the pregnancy, consists in: persistent growth failure, low weight, microcephaly, characteristic facial features, congenital heart diseases (especially septal defects), minor anomalies of joints and limbs, disorders of central nervous system development and function. The complexity, severity and frequency of the anomalies depends on the amount of the ingested alcohol associated or not with other risk factors: low socio-economic level, psychologic disturbances, smoking, drugs. Hearing disorders determined by maternal alcohol consumption, although not classically included in the FAS, and also chronic ear infections, are associated in variable severity degrees. Audiologic procedures performed in FAS children revealed four main hearing disorders: delayed development of auditory function associated with language disorders, sensorineural hearing loss (disorders of middle ear and conductive system), intermittent loss of sound vibrations conduction, hearing loss by central auditory nervous system deficits. Early detection of hearing anomalies alleviates the prognosis by early interventions.
\end{abstract}

Keywords: alcohol, pregnancy, hearing loss

The teratogenic effects of alcohol consumption during pregnancy were described for the first time in the medical literature almost 5 decades ago (1). Jones and Smith in 1973 coined the term fetal alcohol syndrome (FAS) to denote a pattern of birth defects and developmental delays observed in children borned to alcoholic mothers (2).

The prevalence of FAS depends on the studied population, the criteria and assessment method. Thus, in USA the frequency of FAS is estimated at $1-2 / 1.000$ stillborn, while in areas with high risk, like some communities in South Africa, the study of pediatric population revealed an incidence of $4-5 \%$ among the preschool children (3).

Although the maternal alcohol consumption is the main determinant of the negative effects on the fetus, it was demonstrated that also the paternal exposure to alcohol can induce malformations to the fetus. The most frequent clinical features are: facial dismorphism (microcephaly, short palpebral fissures, epicantus, maxilar hypoplasia with aspect of flat face, a hypoplastic philtrum, thin upper lip, micrognathism, cleft palate), insufficient intrauterine or postnatal growth, mental impairment and behav- ioral disorders. Other consequences of alcohol consumption during pregnancy are: spontaneous abortion, premature delivery, intrauterine death, affected bones development and neonatal withdrawal syndrome.

Alterations of gene expression (normal cellular processes in the genome) without disturbing the DNA sequences are known as epigenetic processes. The alcohol disturbes the normal process of cellular differentiation, altering the expression of tissue specific genes. It acts at the level of the nucleosome components, disturbing DNA metilation, histones acetilation and microRNA interactions. Thus, the alcohol is on the list of major factors for epigenetic resets followed by multiorganic malformations (4).

A metaanalysis on 20 studies published between 1987-2013 (5) revealed that the heart malformations occur in $67 \%$ of the children from mothers that consumed alcohol during pregnancy, represented by atrial or ventricular septal defects $21 \%$, Fallot tetralogy and conotruncal defects $6 \%$ and other types of malformations (6). The teratogenic effects depend on the timing, frequency, duration, amount of alcohol exposure as well as the absorp- 
tion and alcohol metabolism by the mother and the fetus, with genetic susceptibility (7).

The cranio-facial malformations are due to the alcohol consumption during organogenesis (18-40 day of pregnancy), while the nervous system disturbancies occur later during pregnancy. After birth, the cognitive disabilities become evident, until severe mental retardation and other psychiatric disorders, like attention deficit, impulsivity and memory troubles. Frequently they associate delays in language acquisition, difficult coordination of movements, behavioral disturbances, low muscle tonus, psychiatric troubles. Otherwise, the prenatal alcohol consumption is the main cause of nonereditary mental retardation (8).

It was advanced that the alcohol prevents the transfer of essential amino acids and zinc from the mother to the fetus, the consequence being the difficulty of protein synthesis and, therefore, growth and development of the fetal handicap (9). Confirmation of alcohol consumption during pregnancy using the biomarkers known in current practice remains a challenge. Recently it has been proposed to include microRNA into profile of SAF investigations.

MicroRNA molecules have a critical role in important biological processes, particularly in utero, ensuring normal development by balancing the balance between their survival and proliferation of cells. In experimental conditions it has been demonstrated that exposure to ethanol supresses some genes involved in regulating organogenesis in various stages of development of the embryo and fetus. There were identified four types of microRNA suppressed by ethanol in neural stem cells (4).

Gardiner and col. noticed that alterations in the levels of specific circulating miRNAs have been associated with various disease states and in animal models of fetal alcohol spectrum disorder and published the results in aprilie 2016. This is the first report demonstrating that serum miRNAs could be useful as biomarkers of alcohol exposure (10).

In the last two decades there were published many studies about the structural changes of the brain determined by alcohol, followed by cognitive and behavioral disabilities both in children with FAS features and especially in those without microcephaly (11).

It was reported the association of FAS with hearing impairment; the commune embryonic origin of the cranio-facial and ocular structures with those that will form the hearing apparatus, favors the association of FAS with hearing impairment.
These structures are derived from the first two branchial arches and have similar periods of sensitivity to exposure to toxic substances. As a result it appears the delay in maturation of auditory components, sensorineural hearing deficiency, central deficiency. These changes are intermittently associated with mechanical type deficiency, consequence of the repeated serous otitis, which these children are prone to. The vestibular damage, relatively common, favorised by the commune embryonic origin, may remain subclinically. Hearing deficiency associated to cognitive deficiency work together, causing a vicious cycle which has consequently delay in language development, language defects and emphasizing cognitive deficit through insufficient stimulation $(12,13)$.

The data accumulated in the past decade have been successful in shaping the four mechanisms that are connected and determine hearing deficiency:

- a developmental delay in auditory maturation;

- sensorineural hearing loss;

- intermittent conductive hearing loss due to recurrent serous otitis media;

- central hearing loss.

The latencies obtained from the primary auditory cortex can represent the amount of latency at all levels of the auditory process, from the moment the sound signal goes through the cochlea, along the nerve flow up to the auditory cortex. Also, the vestibular impairment associated to the auditory impairment, induces a delay in language acquisition, in its characteristics and consequent rise in cognitive impairment (14). Church and coll discovered sensorineural hearing loss in 33\% of children with FAS compared to $2-3 \%$ in the general population, as well as transmission disturbances because of recurrent otitis, which can have a frequency of up to $92 \%$ in FAS group compared with $<20 \%$ in general population (12).

MRI has shown changes in the corpus callosum (15), suggesting risk of atypical hemispheric specialization especially in the area of speech, language and hearing. Perturbation of the interemisferic transfer determine alteration of lateralisation function, children with FAS have presented a large number of errors in the right ear during a listening test, indicating the functional asymmetry (16).

In an animal model of FAS on rats prenatally exposed to different doses of etilic alcohol, the authors observed that almost all the animals showed a peripheral auditory disorder in the form of congen- 
ital sensorineural hearing loss. Alcohol-exposed animals also showed a central auditory processing disorder characterized by prolonged transmission of neural potentials along the brainstem portion of the auditory pathway and also an augmentation in the age-related deterioration of auditory acuity. More, exposure to sounds that can lead to accustic trauma increase the degradation of the sensorial function (17).

A clinical trial reported a significant percentage of $93 \%$ of FAS children with bilateral recurrent serous otitis media, $30 \%$ of them featuring sensorineural hearing losses for which required mechanical sound amplification. Also, the eustachian tube dysfunction coupled with immune deficiencies predispose these children at recurrent upper respiratory tract infections. Possible alcohol ototoxicity and an alcohol-induced neuroectoderm syndrome explains the typical hearing impairment within the FAS, although this component wrongly is not yet recognized as one of the elements characteristic of the syndrome. Accepting this idea would broaden the therapeutic scheme in the diagnosed patients and may lead to an improvement in psychological, medical and social prognosis (13).

\section{REFERENCES}

1. Lemoine P., Harouseau B., Borteryu J.T., Menuet J.C. Les enfants des parents alcooliques: Anomalies observees apropos de 127 cas. Ouest Medical. 1968; 21:476-482.

2. Jones K.L., Smith D.W. Recognition of the fetal alcohol syndrome in early infancy. Lancet. 1973; 302:999-1001.

3. May P.A., Brooke L., Gossage J.P., et al. Epidemiology of fetal alcohol syndrome in a South African community in the Western Cape Province. Am J Public Health. 2000 Dec. 90(12):1905-12.

4. Ungerer Knezovich J., Ramsey M. In utero alcoholic exposure, epigenetic changes, and their consequences. Alcohol Res 2013; 35(1):37-46.

5. Yang J., Qiu H., Qu P., Zhang R., Zeng L., Yan H. Prenatal Alcohol Exposure and Congenital Heart Defects: A Meta-Analysis., PLoS One. 2015 Jun 25;10(6).

6. Burd L., Deal E. et al. Congenital heart defects and fetal alcohol spectrum disorders, Congen Heart Dis 2007, 2 (4): 250-255.

7. Ungerer M., Knezovich J., Ramsay M. In utero alcohol exposure, epigenetic changes, and their consequences. Alcohol Res. 2013; 35(1):37-46.

8. Gray R., Mukherjee R.A., Rutter M. Alcohol consumption during pregnancy and its effects on neurodevelopment: what is known and what remains uncertain. Addiction. 2009 Aug. 104(8):1270-3.

9. Robert M. Kliegman M.D. Nelson Textbook of Pediatrics, 20 th Edition, Volume 1, Elsevier, 2016, p. 895.

10. Gardiner A.S., Gutierrez H.L. et al. Alcohol use during pregnancy is associated with specific alterations in microARN levels in maternal serum, Alcohol Clin Exp Res 2016, 40 (4): 826-837.
Auditory delay revealed by magnetoencephalography in children with FAS may prove to be a useful neural marker of information processing difficulties in young children with prenatal alcohol exposure. The fact that delayed auditory responses were observed across the FAS spectrum suggests that it may be a sensitive measure of alcohol-induced brain damage. Therefore, the authors propose to include this investigation in conjunction with other clinical tools for early identification of alcohol affected children, particularly those without dysmorphia (18).

\section{CONCLUSIONS}

SAF, whose severity depends on the precocity and abundance of alcohol consumption, often associates hearing disorders. Alcohol toxicity is delaying the maturation of structures intended for hearing, affects the sensorineural and central type auditory component, creates problems in the transmittion of sound. Hearing loss, included as part of the diagnosis of the FAS must be detected early, treated properly for a good development of language, improving the quality of life and better psycho-social integration.

11. Bookstein F.L., Streissguth A.P. et al. Damage to the human cerebellum from prenatal alcohol exposure: the anatomy of a simple biometrical explanation, Anat Rec B New Anat 2006, 289: 195-209.

12. Church M.W., Kaltenbach J.A. Hearing, Speech, Language, and Vestibular Disorders in the Fetal Alcohol Syndrome: A Literature Review, Alcohol Clin Exp Res 1997, 21 (3): 495-512.

13. Church M.W., Gerkin K.P. Hearing disorders in children with fetal alcohol syndrome: findings from case reports, Pediatrics 1988; 82 (2): 147-154.

14. Church M.W., Abel E.L. Fetal alcohol syndrome. Hearing, speech, language, and vestibular disorders, Obstet Gynecol Clin North Am. 1998, 25(1):85-97.

15. Bookstein F.L., Sampson P.D., Connor P.D., \& Streissguth, A.P. (2002). Midline corpus callosum is a neuroanatomical focus of fetal alcohol damage. Anatomical Record, 269, 162-174.

16. Domellof E., Ronnqvist L., Titran M., Esseily R., \& Fagard J. Atypical lateralization in children with fetal alcohol syndrome, Developmental Psychobiology 2009, 51:696-705.

17. Church M.W., Abel E.L., Kaltenbach J.A., Overbeck G.W. Effects of prenatal alcohol exposure and aging on auditory function in the rat: preliminary results, Alcohol Clin Exp Res. 1996, 20(1): 172-179.

18. Stephen J.M., Kodituwakku P.W. et al. Delays in auditory processing identified in preschool children with FASD, Alcohol Clin Exp Res 2012, 36(10): 1720-1727. 